\title{
The detector system of the Muon Ionization Cooling Experiment (MICE) experiment
}

\author{
MAURIZIO BONESINI ${ }^{* \dagger}$ \\ Sezione INFN Milano Bicocca \\ E-mail: maurizio.bonesini@mib.infn.it
}

The International Muon Ionization Cooling Experiment (MICE) at RAL aims at a demonstration of ionization cooling, for application in future neutrino factories or muon colliders. Beam emittances are measured with an absolute precision of $0.1 \%$ on a single particle basis via a sophisticated beam instrumentation system. This is made of two trackers inside spectrometer solenoids and a particle identification system, based on three time-of-flight stations, two Cherenkov detectors and a downstream calorimeter. Some results obtained in MICE STEP I (the beamline characterization) will be shown, demonstrating the good performances of the installed beam instrumentation.

The European Physical Society Conference on High Energy Physics 18-24 July, 2013

Stockholm, Sweden

\footnotetext{
*Speaker.

$\dagger$ on behalf of the MICE Collaboration
} 
The MICE experiment [1] at RAL aims at a systematic study of a section of the US Study 2 [2] cooling channel, attaining a $10 \%$ emittance reduction for a $6 \pi \cdot \mathrm{mm}$ rad, $200 \mathrm{MeV} / \mathrm{c}$ beam. The 5.5 $\mathrm{m}$ long cooling section consists of three liquid hydrogen absorbers (AFC) and eight $201 \mathrm{MHz}$ RF cavities surrounded by lattice solenoids. As conventional emittance measurement techniques, reach

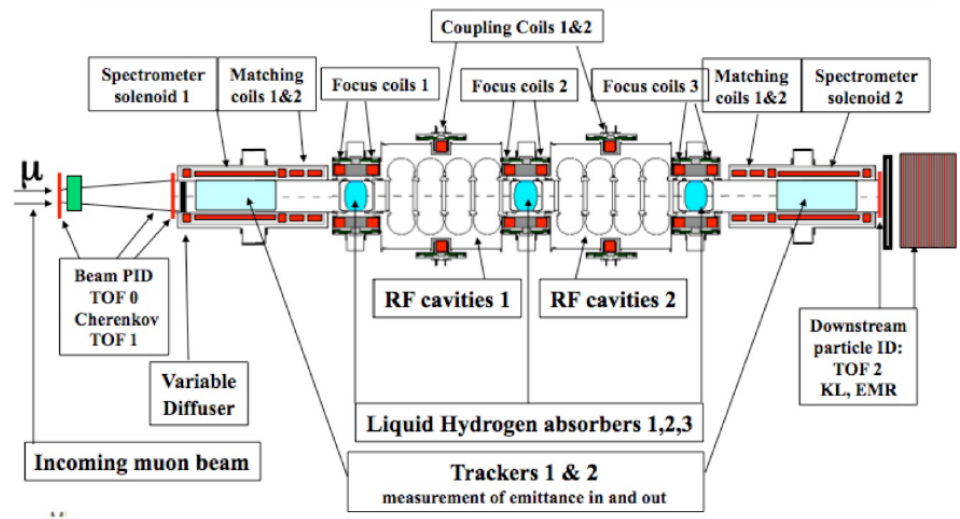

Figure 1: View of the MICE experiment at RAL. The muon beam from ISIS (140-240 MeV/c, tunable between $3-10 \pi \cdot \mathrm{mm}$ rad input emittance) enters from the left. The cooling channel is between two magnetic tracking spectrometers and two TOF stations (TOF1 and TOF2) that measure particle parameters.

barely a $\sim 10 \%$ precision, a novel method based on the measurement of $x, y, t, p_{x}, p_{y}, E$ coordinates for individual beam particles, before and after the cooling section, has been proposed [1].

The experiment will be done in several steps, of which the first one (STEP I) is the characterization of the beamline. The next STEP IV will aim instead to measure the cooling effects in different configurations.

MICE is equipped with a system for particle identification, based on a time-of-flight (TOF) system, two Cherenkov detectors, a downstream calorimeter (KL + EMR) and two scintillating fiber trackers within spectrometer solenoids (see figure 1). The trackers will measure the spatial coordinates, angles and momenta $\left(x, y, x^{\prime}, y^{\prime}, p\right)$, of each particle with a resolution $\sim 10 \%$ of the phase space equilibrium emittance width in each dimension. A single tracker station was tested in MICE beam line and a full tracker module (5 stations) with cosmics showing good performances, see reference [3] for more details. The EMR is a fully active calorimeter and will be used to measure the muon range in the downstream section of the apparatus, allowing complementary information on their momentum. Details of its construction are summarized in reference [4].

To date, the beamline characterization (STEP I) used informations from only the TOF and KL systems. The remaining detectors will be used in step IV.

The TOF system includes three time-of-flight detectors, made of fast Bicron scintillator, read out at both ends by conventional Hamamatsu R4998 photomultipliers. They have intrinsic detector resolutions $\sigma_{t} \sim 50 \mathrm{ps}$ and are described in reference [5]. The KL is a Pb-scintillating fiber calorimeter of the KLOE type [6], with active area $93 \times 93 \mathrm{~cm}^{2}$, to tag electrons from downstream $\mu$ decays. In STEP I the following results were obtained:

- characterization and optimization of MICE beamline [7]

- a preliminary measurement of the beamline emittance with the TOF system only [8] 
- the measurement of the pion contamination of the MICE muon beam [9]

The TOF system and the KL calorimeter performed to expectation. For example, they were used to measure the pion contamination in the MICE muon beam. Calibration runs at different momenta were used to define time-of-flight windows with a dominant pion or muon content (see left panel of figure 2). The samples were used to define pion or muon templates ( AKA a pattern of energy deposition in KL), which were in turn fitted to muon runs to determine their pion contamination (see the right panel of figure 2).
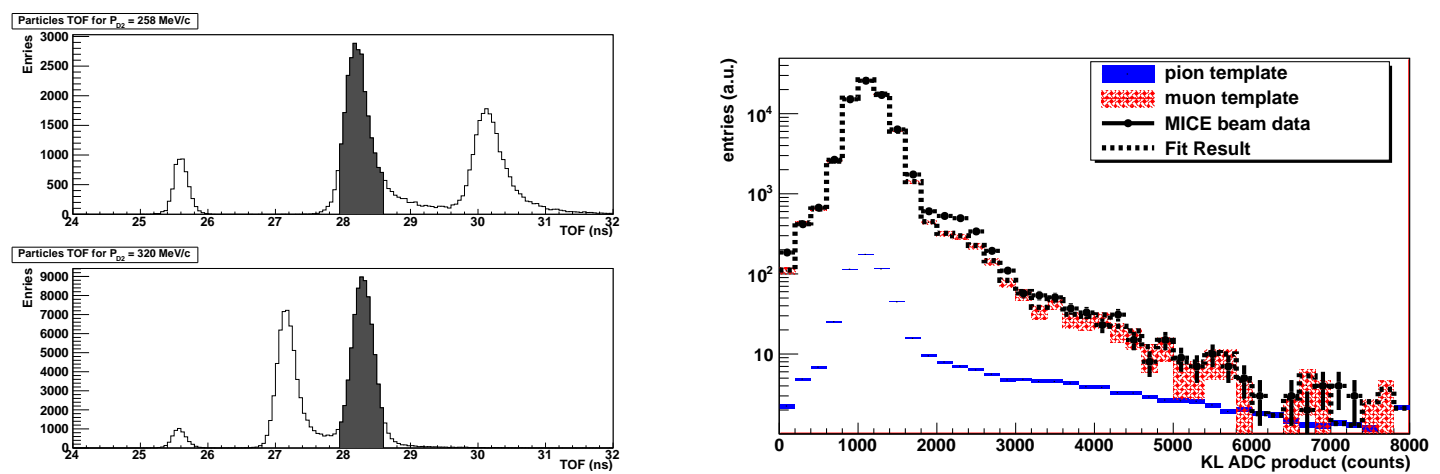

Figure 2: Left panel: Time-of-flight distributions in two paired calibration beam settings at 258 and 320 $\mathrm{MeV} / c$. The interval 28.0-28.6 ns (shaded) is populated by muons (pions) in upper (lower) plot. Right panel: MICE beam data (black dots), muon (red dotted area) and pion (blue solid area) fractions, are normalised to the the template fit (black histogram) performed to the KL ADC product spectrum.

Using this method, the pion contamination was computed as: $(1.11 \pm 0.29 \pm 0.32) \%$, in agreement with Monte Carlo estimates (see reference [9] for more details). The measured pion contamination translates to a pion contamination of $(0.82 \pm 0.22 \pm 0.24) \%$ at the entrance of the MICE cooling channel (first Focus coil $\sim 3.36 \mathrm{~m}$ downstream of TOF1). This value is well within the contamination requirements for the muon MICE beamline.

\section{References}

[1] G. Gregoire et al., MICE Proposal to RAL, 2003.

[2] S.Ozaki et al., BNL-52623, 2001 ; M.M. Alsharo'a et al., Phys. ReV. ST. Accel. Beams 6,081001 (2003); R. Palmer et al., arXiv:0711.4275.

[3] E. Overton, proceedings VCI 2013, Vienna, to be published on Nucl. Instr. and Meth. ; M. Ellis et al. Nucl. Instr. Meth. A659 (20110 156.

[4] R.Asfandiyanov, proceedings VCI 2013, Vienna, to be published on Nucl. Instr. and Meth.

[5] R. Bertoni et al., Nucl. Instr. and Meth. A615 (2010) 14; M. Bonesini et al. Nucl. Instr. and Meth. A693 (2012) 130.

[6] A. Aloisio et al. Nucl. Instr. and Meth. A494 (2002) 326

[7] M.Bogomilov et al., JINST 7 (2012) P05009.

[8] D. Adams et al. Eur. Phys. J. C73 (2013) 2582.

[9] M. Bogomilov et al., MICE note 416 (2013) 